\title{
Comparative Evaluation of GeneXpert MTB/RIF Assay and Microscopy for Rapid Diagnosis of Tuberculous Meningitis in Children
}

\author{
Shweta Chitkara*, Ravinder Kaur, Yogita Rai and Gaurav Dhaka \\ Department of Microbiology, Lady Hardinge Medical College, Connaught Place, \\ New Delhi- 110001, India \\ *Corresponding author
}

\section{A B S T R A C T}

Keywords

Drug resistance;

GeneXpert;

Meningitis;

Tuberculosis

Article Info

Accepted:

05 February 2020

Available Online:

10 March 2020
Tuberculous meningitis (TBM) is the most devastating complication of Mycobacterium tuberculosis infection. Diagnosis of TBM is challenging in young children due to the paucibacillary nature of disease. Microscopy for acid-fast bacilli in CSF is fast but has very low sensitivity whereas culture may take up to 42 days. Due to the urgency of diagnosis in suspected TBM cases, a rapid, accurate diagnostic test could have a great impact on survival. Aims of the study are to prospectively determine the diagnostic accuracy of Xpert MTB/RIF in a large consecutive series of samples from patients presenting with suspected Tuberculous meningitis. In this prospective hospital-based study, 147 children presenting with suspected tuberculous meningitis from May 2017 to February 2018 were included. Cerebrospinal fluid samples were tested by Ziehl-Neelsen smear, mycobacterial culture on Lowenstein Jensen medium, and Xpert MTB/RIF assay. Kappa statistics $(\mathrm{K})$ was used to determine agreement beyond what would be expected by chance between smear microscopy and Gene Xpert assay results. Out of a total of 147 CSF samples tested, $10(6.8 \%)$ were positive for Mycobacterium tuberculosis by Xpert MTB/RIF assay. Out of these 10 samples, only one showed AFB on ZN smear. Culture was negative for all the $147 \mathrm{CSF}$ samples. The MTB/RIF test has a short turnaround time and simultaneously detects $M$. tuberculosis and RIF resistance in less than $2 \mathrm{~h}$. It could be a useful tool for rapid identification of M. tuberculosis, especially in smear-negative clinical samples.

\section{Introduction}

Tuberculous meningitis (TBM) is the most devastating consequence of infection with Mycobacterium tuberculosis. Approximately a third of patients die soon after presenting to hospital, and many of those surviving are left with severe neurological sequelae. ${ }^{(1,2)}$ Rapid TBM diagnosis and treatment is a strong prognostic indicator for reduced death and neurologic deficit.

However, many patients are diagnosed late because initial signs are non-specific, and rapid and sensitive diagnostic tests are lacking. ${ }^{(3)}$

Available TBM diagnostic tests are inadequate, for the most part due to low 
bacillary loads in CSF. ${ }^{(4)}$ Ziehl-Neelsen (ZN) microscopy staining of cerebrospinal fluid (CSF) is the most widely applied rapid diagnostic technique; however, sensitivity for TBM rarely exceeds $20 \%$. ${ }^{(5)}$ Liquid culture techniques, including the mycobacterial growth indicator tube (MGIT; Bactec) and the mycobacterial observation drug susceptibility assay (MODS) culture offer improved sensitivity over solid culture, to a sensitivity of almost $60 \%{ }^{(6)}$ The clinical value of culture techniques is limited to diagnostic confirmation and drug susceptibility testing, because they take 1 to 4 weeks to return a positive result, and negative results cannot be used to exclude a TBM diagnosis.

The GeneXpert MTB/RIF test (Cepheid) is a closed-cartridge based system that is easy to operate by minimally trained staff and gives results in approximately2hours. ${ }^{(7)}$ The Xpert MTB/RIF test was approved by the WHO in 2010 for the diagnosis of pulmonary TB following extensive evaluation projects in six countries led by the Foundation for Innovative New Diagnostics (FIND). ${ }^{(8)}$

The test is based on a real-time heminested PCR test which detects the presence of $M$. tuberculosis complex bacilli. ${ }^{(9)}$ By using 5 molecular beacons which span the rpoB gene 81-bp rifampin resistance-determining region (RRDR), the test simultaneously determines susceptibility to rifampin, which can be used as a surrogate marker for multidrug resistance (MDR) ${ }^{(9)}$ The closed-cartridge system makes it possible for the assay to be used outside the laboratory environment, and studies assessing biosafety have suggested that the use of Xpert MTB/RIF carries a smaller biohazard risk than smear microscopy. ${ }^{(7)}$ The risk of crosscontamination is also reduced with the closed cartridge system. $^{(7)}$ The test has shown sensitivity above $90 \%$ for culture positive tuberculosis, with high specificity in sputum samples. Sensitivity in individuals with HIV coinfection is over $80 \% \cdot{ }^{(10-12)}$ Several studies have reported successful use of the Xpert MTB/RIF test on extrapulmonary samples, with overall sensitivities of over $80 \%$ and specificity reaching $100 \% .^{(13-17)}$

Due to the urgency of diagnosis in suspected TBM cases, a rapid, accurate diagnostic test which also is able to identify rifampin resistance could have a great impact on survival. The aim of the present study was to prospectively determine the diagnostic accuracy of Xpert MTB/RIF as compared to smear microscopy in a large consecutive series of samples from patients presenting to the hospital with suspected tuberculous meningitis.

\section{Materials and Methods}

In this prospective hospital-based study, 147 children presenting with suspected tuberculous meningitis from May 2017 to February 2018 were included. A clinical diagnosis of meningitis was made based on clinical findings, CSF criteria or both. ${ }^{(18)}$

Clinical criteria of meningitis included headache, fever and neck stiffness, with or without altered consciousness.

CSF criteria were cell count $>10$ cell $/ \mathrm{mm} 3$, protein concentration $>45 \mathrm{mg} / \mathrm{dL}$, or the CSF:blood glucose ratio <0.5; either alone or in combination.

Signs and symptoms were recorded using standardized forms, and CSF was obtained by lumbar puncture for routine examination and microbiological testing.

All samples were subjected to Ziehl-Neelsen (ZN) staining, Xpert MTB/RIF (Cepheid, Sunnyvale, US) assay and culture inoculation. All samples were processed in level II biosafety cabinet. 


\section{Ziehl-Neelsen smear}

Ziehl-Neelsen smears were prepared using standard methods. The layered smear was then stained according to standard procedures. The ZN smear was meticulously examined under a 1,000 magnification before being recorded as negative. Observation of a single acid-fast bacillus was considered a positive result. $^{(19)}$

\section{Solid culture on LJ medium}

Sterile, uncentrifuged CSF samples were directly inoculated without decontamination. A slope of LJ medium was inoculated with 0.25 to $0.5 \mathrm{~mL}$ of each specimen and incubated at $37^{\circ} \mathrm{C}$ for eight weeks. The LJ slants were inspected weekly. Growth on the LJ slants resembling mycobacterial colonies i.e., rough, tough and buff-coloured, was subjected to ZN staining and Gram staining to confirm the presence of acid-fast bacilli and rule out contamination respectively.

\section{Xpert MTB/RIF}

The Xpert MTB/RIF test was performed using the G4 version of cartridges as per the manufacturer's instruction (Cepheid, Sunnyvale, CA). Unprocessed samples were used directly for performing the test and no frozen samples were used in the study. The sample was mixed with the sample reagent supplied with the test (2:1 ratio) and left to stand for $15 \mathrm{~min}$, as per the manufacturer's instructions, with intermittent manual shaking. The solution was then transferred to the Xpertcartridge using a provided pipette, and the cartridge was loaded onto the Xpert machine for analysis. Results were reported as positive or negative for $M$. tuberculosis. Positive results were placed in one of four categories; very low, low, medium, or high. Rifampin resistance results were reported as susceptible or resistant. ${ }^{(20)}$

\section{Analysis}

Statistical analysis was performed using SPSS version 22 (IBM Inc., Armonk, NY). Kappa statistics (K) was used to determine agreement beyond what would be expected by chance between smear microscopy result and Gene Xpertmtb/rif assay results. Four levels of agreement for kappa: <0.40 (poor), 0.40 0.59 (fair), $0.60-0.80$ (good), and $>0.80$ (excellent) were reported.

\section{Results and Discussion}

A total of 147 consecutive patients with suspected meningitis were included. Patients were mostly male $(60 \%)$, with a median age of 6 years(range 0.5-16 years), presenting after a median of 14 days (range 7-30 days) of symptoms, mostly with fever $(100 \%)$, headache $(67 \%)$ and altered consciousness $(31.3 \%)$ as chief complaints. On examination, nuchal rigidity (77.2\%), lowered consciousness (52.6\%), and focal neurological signs were common.

Out of a total of 147 CSF samples tested, 10 (6.8\%) were positive for Mycobacterium tuberculosis by GeneXpert MTB/RIF assay. None showed rifampicin resistance. Out of these 10 samples, only one showed AFB on $\mathrm{ZN}$ smear. Culture was negative for all the 147 CSF samples (Figure 1).

A comparison of smear microscopy and GeneXpert was done to gauge the concordance of microscopy results and GeneXpert MTB/RIF results, the findings revealed a significant $(\mathrm{p}=0.003)$ variation in agreement between the two assays $93.8 \%$ with a Cohen's kappa, 0.172(CI=-0.122 0.465) (Table 1).

In this study, performance of the GeneXpert MTB/RIF assay with CSF specimens of patients who were suspected as having tubercular meningitis was evaluated. 
Table.1 Kappa score on reliability of smear microscopy and GeneXpert assay

\begin{tabular}{|c|c|c|c|c|c|c|}
\hline \multicolumn{7}{|c|}{ GeneXpert MTB/RIF assay } \\
\hline $\begin{array}{c}\text { Smear } \\
\text { microscopy }\end{array}$ & $\begin{array}{c}\text { Positive } \\
\mathbf{N}(\%)\end{array}$ & $\begin{array}{c}\text { Negative } \\
\mathbf{N}(\%)\end{array}$ & Agreement & Kappa & $95 \% \mathbf{C l}$ & p-value \\
\hline Positive & 1 & 0 & 93.8 & 0.172 & $\begin{array}{c}-0.122- \\
0.465\end{array}$ & 0.003 \\
\hline Negative & 9 & 137 & & & & \\
\hline
\end{tabular}

Fig.1 Venn diagram of overlap in TB meningitis diagnostics

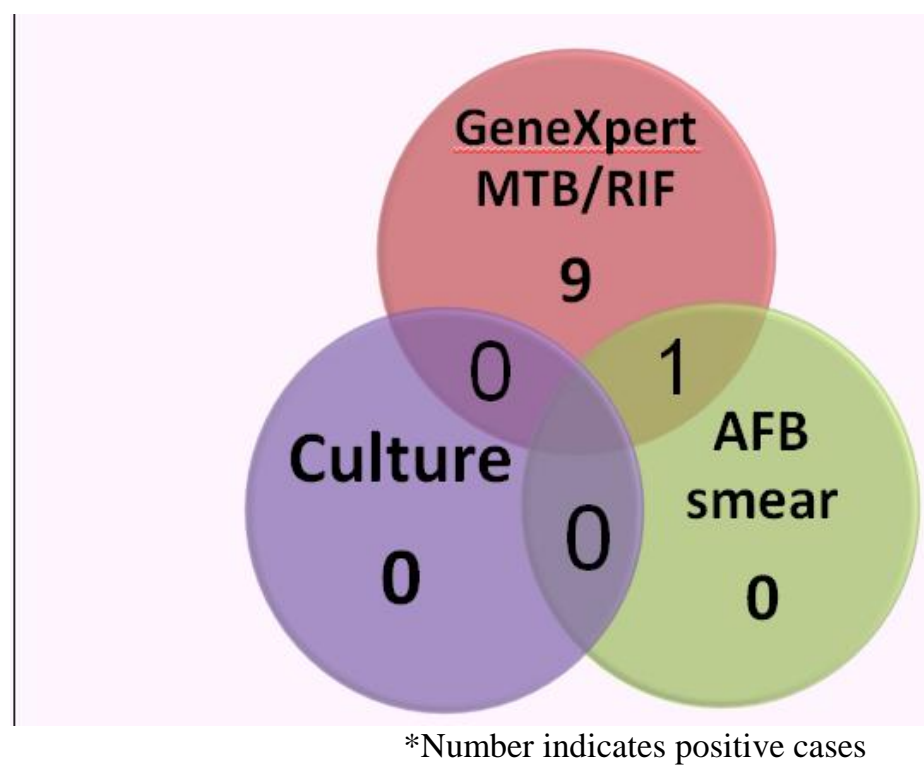

The positive rate was $6.8 \%$ by GeneXpert $\mathrm{MTB} / \mathrm{RIF}$, and this rate was higher than that $(0.68 \%)$ by a direct AFB smear examination. Our finding that the $\mathrm{ZN}$ smear is less sensitive than the GeneXpert MTB/RIF test is reasonable because the $\mathrm{ZN}$ smear method requires $5 \times 10^{3}$ to $1 \times 10^{4}$ bacilli/ml of specimen to generate a positive result. ${ }^{(21)}$ The out performance of Xpert MTB/RIF detected in current work is in agreement with other researchers who established the diagnosis in a significant proportion of patients and up to $10-15 \%$ of smear-negative TB. ${ }^{(22,23)}$

In this study, culture was negative for all the 147 CSF samples, may be due to less number of viable M.tuberculosis in CSF samples.

The average turnaround time (including time taken to process specimens and testing time) 
of the GeneXpert MTB/RIF assay and ZN smear method was significantly shorter $(2.5$ and 3.5 hours) than that of the solid culture on LJ medium (42 days). A shorter turnaround time can help TB patients be diagnosed early and treated in time. Therefore, the GeneXpert MTB/RIF assay has an obvious advantage in management of TB.

In our National TB Control Program (RNTCP), CBNAAT is the preferable investigation of choice for diagnosis of pediatric tuberculosis, especially in cases of extrapulmonary TB. ${ }^{(24)}$

Although GeneXpert assay is considered a breakthrough in the diagnosis of TB and extra pulmonary TB (EPTB), one of the major limitations of this technique is that it cannot distinguish between viable and non-viable microorganisms while detecting mycobacterial DNA. A study by Boyles et al., on previously treated tuberculosis cases showed false positivity of CBNAAT due to dead bacilli. ${ }^{(25)}$

The GeneXpert MTB/RIF assay is a simple, rapid, and accurate test method for detecting M.tuberculosis, is less dependent on the operator's skills, and staff with minimal training can use the equipment. Although the GeneXpert MTB/RIF assay has these advantages, similar to other tests for M.tuberculosis, a negative result can not exclude the diagnosis of TB, and patients with positive results can also be assessed comprehensively with results of the $\mathrm{ZN}$ smear test, culture, clinical symptoms, and radiographic evidence.

In conclusion, the MTB/RIF test has a short turnaround time and simultaneously detects M. tuberculosis and RIF resistance in less than 2hours. It could be a useful tool for rapid identification of $M$. tuberculosis, especially in smear-negative clinical samples. Further studies withculture or predefined CRS (certified reference standard)with follow up are required to define the exact sensitivityand specificity of GeneXpert.

\section{References}

1. Brancusi F, Farrar J, Heemskerk D. 2012. Tuberculous meningitis in adults: a review of a decade of developments focusing on prognostic factors for outcome. Future Microbiol. 7:1101-1116. http://dx.doi.org/10. 2217/fmb.12.86.

2. Thwaites GE. 2013. Advances in the diagnosis and treatment of tuberculous meningitis. Curr. Opin. Neurol. 26:295$300 . \quad$ http://dx.doi.org/10 .1097/WCO.0b013e3283602814.

3. Hosoglu S, Geyik MF, Balik I, Aygen B, Erol S, Aygencel TG, et al., 2002. Predictors of outcome in patients with tuberculous meningitis. Int. J. Tuberc. Lung Dis. 6:64 -70.

4. Ho J, Marais BJ, Gilbert GL, Ralph AP. Diagnosing tuberculous meningitis - have we made any progress? Trop Med Int Health 2013; 18:783-93.

5. Garg RK. 1999. Tuberculosis of the central nervous system. Postgrad. Med. J. 75:133-140.

6. Caws M, Dang TM, Torok E, Campbell J, Do DA, Tran TH, et al., 2007. Evaluation of the MODS culture technique for the diagnosis of tuberculous meningitis. PLoS One 2:e1173. http://dx.doi.org/10.1371/journal.pone.00 01173.

7. Lawn SD, Nicol MP. 2011. Xpert(R) MTB/RIF assay: development, evaluation and implementation of a new rapid molecular diagnostic for tuberculosis and rifampicin resistance. Future Microbiol. 6:1067-1082. http://dx.doi.org/10.2217/fmb.11.84.

8. World Health Organization. 2011. Automated real time nucleic acid 
amplification technology for rapid and simultaneous detection of tuberculosis and rifampicin resistance: Xpert MTB/RIF system. Policy statement. WHO/HTM/TB/2011.4. World Health Organization, Geneva, Switzerland.

9. Boehme CC, Nabeta P, Hillemann D, Nicol MP, Shenai S, Krapp F, et al., 2010. Rapid molecular detection of tuberculosis and rifampin resistance. $\mathrm{N}$. Engl. J. Med. 363:1005-1015. http://dx.doi.org /10.1056/NEJMoa0907847.

10. Lawn SD, Brooks SV, Kranzer K, Nicol MP, Whitelaw A, Vogt M, et al., 2011. Screening for HIV-associated tuberculosis and rifampicin resistance before antiretroviral therapy using the Xpert MTB/RIF assay: a prospective study. PLoS Med.8:e1001067.http://dx.doi .org/10.1371/journal.pmed.1001067.

11. Scott LE, McCarthy K, Gous N, Nduna M, Van Rie A, Sanne I, et al., 2011. Comparison of Xpert MTB/RIF with other nucleic acid technologies for diagnosing pulmonary tuberculosis in a high HIV prevalence setting: a prospective study. PLoS Med. 8:e1001061. http: //dx.doi.org/10.1371/journal.pmed.10010 61.

12. Theron G, Peter J, Van Zyl-Smit R, Mishra H, Streicher E, Murray S, et al., 2011. Evaluation of the Xpert MTB/RIF assay for the diagnosis of pulmonary tuberculosis in a high HIV prevalence setting. Am. J. Respir. Crit. Care Med. 184:132-140.

http://dx.doi.org/10.1164/rccm.201101 0056OC

13. Lawn SD, Zumla AI. 2012. Diagnosis of extrapulmonary tuberculosis using the Xpert((R)) MTB/RIF assay. Expert Rev. Anti Infect. Ther. 10: 631- 635. http://dx.doi.org/10.1586/eri.12.43.

14. Tortoli E, Russo C, Piersimoni C,
Mazzola E, Dal MP, Pascarella M, et al., 2012. Clinical validation of Xpert MTB/RIF for the diagnosis of extrapulmonary tuberculosis. Eur. Respir. J. 40:442- 447 . http://dx.doi .org/10.1183/09031936.00176311.

15. Hillemann D, Rusch-Gerdes S, Boehme C, Richter E. 2011. Rapid molecular detection of extrapulmonary tuberculosis by the automated GeneXpert MTB/RIF system. J. Clin. Microbiol. 49:1202-1205. http://dx .doi.org/10.1128/JCM.02268-10.

16. Moure R, Martin R, Alcaide F. 2012. Effectiveness of an integrated real-time PCR method for detection of the Mycobacterium tuberculosis complex in smear-negative extrapulmonary samples in an area of low tuberculosis prevalence. J. Clin. Microbiol. 50:513-515. http://dx.doi.org /10.1128/JCM.06467-11.

17. Vadwai V, Boehme C, Nabeta P, Shetty A, Alland D, Rodrigues C. 2011. Xpert MTB/RIF: a new pillar in diagnosis of extrapulmonary tuberculosis? J. Clin. Microbiol. 49:2540-2545. http://dx.doi.org/10.1128/JCM.02319-10.

18. Forbes BA, Sahm DF, Weissfeld AS, editors. 11th ed. St. Louis: The CV Mosby company; 2002. Meningitis and other infections of Central Nervous system, chapter 58 In: Bailey and Scott's Diagnostic Microbiology; p. 907.

19. Thwaites GE, Chau TT, Farrar JJ. 2004. Improving the bacteriological diagnosis of tuberculous meningitis. J. Clin. Microbiol. $\quad 42: 378 \quad-379$. http://dx.doi.org/10.1128/JCM.42.1.378379.2004.

20. Xpert MTB RIF kit insert. Available from http://www.cepheid.com/manageddownlo ads/xpert-mtb-rif-english-package-insert301-1404-rev-b-february-2015.pdf.

21. Diagnostic standards and classification of tuberculosis in adults and children. Am. J. Respir. Crit. Care Med 2000; 161(4 Pt 1): 1376-1395. 
22. G. Theron, J. Peter, Z.R. Van, H. Mishra, E. Streicher, S. Murray, et al., Evaluation of the Xpert MTB/RIF assay for the diagnosis of pulmonary tuberculosis in a high HIV prevalence setting Am. J. Resp. Crit. Care Med., 184 (1) (2011), pp. 132140

23. S. Iram, Hussain S, ZeenatA, N.W. Yusuf, M. Aslam. Rapid diagnosis of tuberculosis using Xpert MTB/RIF assayreport from a developing country Pak. J. Med. Sci., 31 (1) (2015), pp. 105-110

24. Revised National TB Control Programme.
Technical Guidelines for Tuberculosis Control in India 2016, Central TB Division, Director General of Health Service, Ministry of Health \& Family Welfare, India. www.tbindia.gov.in.

25. Boyles TH, Hughes J, Cox V, Burton R, Meintjes G, Mendelson M. False-positive Xpert ${ }^{\circledR}$ MTB/RIF assays in previously treated patients: need for caution in interpreting results. The International Journal of Tuberculosis and Lung Disease. 2014 Jul 1;18(7):876-8.

\section{How to cite this article:}

Shweta Chitkara, Ravinder Kaur, Yogita Rai and Gaurav Dhaka. 2020. Comparative Evaluation of GeneXpert MTB/RIF Assay and Microscopy for Rapid Diagnosis of Tuberculous Meningitis in Children. Int.J.Curr.Microbiol.App.Sci. 9(03): 25-31. doi: https://doi.org/10.20546/ijcmas.2020.903.004 